\title{
Studies of Potassium Tungsten Bronzes Containing Lithium or Sodium
}

\author{
Altaf Hussain* and Lars Kihlborg ${ }^{\S}$ \\ Department of Inorganic Chemistry, Arrhenius Laboratory, University of Stockholm, S-10691 Stockholm, \\ Sweden
}

\begin{abstract}
Hussain, A. and Kihlborg, L., 1987. Studies of Potassium Tungsten Bronzes Containing Lithium or Sodium. - Acta Chem. Scand., Ser. A 41: 18-23.

The systems $\mathrm{M}_{y} \mathrm{~K}_{x} \mathrm{WO}_{3}$ with $\mathrm{M}=\mathrm{Li}$ or $\mathrm{Na}$ have been prepared from oxide mixtures in closed ampoules at $500-850^{\circ} \mathrm{C}$ and subsequently examined by X-ray diffraction, electron diffraction and scanning electron microscopy. Intergrowth tungsten bronze (ITB) containing significant amounts of $\mathrm{Li}$ or $\mathrm{Na}$ could not be prepared. Hexagonal tungsten bronze (HTB) forms with $\mathrm{Li}$ for $x>0.19$ and $(x+y)<0.33$ but does not form with Na. A tetragonal tungsten bronze (TTB) phase was obtained with both $\mathrm{Li}$ and $\mathrm{Na}$ for $x>0.45$ and $(x+y)<0.6$. The lithium-containing phases were found to be unstable in aqueous environment, Li being leached out.
\end{abstract}

The alkali tungsten bronzes are well-known nonstoichiometric compounds represented by the general formula $\mathrm{A}_{x} \mathrm{WO}_{3}$, where $\mathrm{A}$ is typically an alkali metal. There are four main structural types, namely (i) intergrowth tungsten bronze, ITB, (ii) hexagonal tungsten bronze, HTB, (iii) tetragonal tungsten bronze, TTB, and (iv) perovskite tungsten bronze, PTB. The ITB and HTB bronzes form only with $\mathrm{K}, \mathrm{Rb}$ or Cs, ITB for $x=$ approx. $0.06-0.10$ and HTB for $x=0.19-0.33$. $^{1}$ TTB can be obtained with $\mathrm{Na}$ and $\mathrm{K}$, with $x$ values in the range $0.28-0.48$ for $\mathrm{Na}$ and $0.42-$ 0.57 for $\mathrm{K} .{ }^{1} \mathrm{~A}$ review of the conditions of formation and structures of these phases has been published earlier. ${ }^{2}$

It is of interest to know to what extent the smaller alkali metals can be incorporated into these phases. Mixed alkali metal tungsten bronzes, $\mathbf{M}^{\prime}{ }_{x} \mathbf{M}^{\prime \prime} \mathrm{WO}_{3}$, where $\mathbf{M}^{\prime}$ and $\mathbf{M}^{\prime \prime}$ are two different alkali metals and $x+y<1$, have been studied previously. Banks and Goldstein ${ }^{3}$ showed that lithium could be incorporated in the K-HTB phase and reported that its homogeneity range with respect to total alkali was thereby extended

\footnotetext{
* On leave from Dept. of Chemistry, Dhaka University, Dhaka-2, Bangladesh.

$\$$ To whom correspondence should be addressed.
}

to $0.13<(x+y)<0.51$. Utsumi and Fujieda prepared a series of $\mathrm{Na}_{y} \mathrm{~K}_{x} \mathrm{WO}_{3}$ TTB phases by electrolysis of tungstate melts ${ }^{4}$ and Fujieda studied their optical properties. ${ }^{5}$ Drobasheva et al. have prepared mixed $\mathrm{Na}-\mathrm{Cs}, \mathrm{K}-\mathrm{Cs}$ and $\mathrm{K}-\mathrm{Rb}$ bronzes, also by melt electrolysis, but the phases obtained are poorly characterized. ${ }^{6-8}$ Intercalation of lithium into K-HTB by electrochemical means at ambient temperature or by using organic lithium compounds has also been reported. ${ }^{-11}$

We have investigated the possibility of obtaining mixed lithium-doped ITB bronzes and in connection herewith also reinvestigated the corresponding HTB and TTB phases. Some experiments with sodium doping were also made. The results are presented below.

\section{Experimental}

Potassium tungstate (BDH; reagent grade), sodium tungstate (Merck; reagent grade), tungsten trioxide (Koch-Light Labs. Ltd.; 99.9\%) and tungsten dioxide (ICN Pharmaceuticals; reagent grade) were commercially available. Lithium tungstate was prepared by heating a mixture of lithium carbonate (Baker; reagent grade) and tungsten trioxide in air in a gold crucible, first at 
$500^{\circ} \mathrm{C}$ for $3-4$ days and then at $650^{\circ} \mathrm{C}$ for a week. The phase purity of these reactants was checked by X-ray powder diffraction.

Bronzes $\mathrm{M}_{y} \mathrm{~K}_{x} \mathrm{WO}_{3}$, where $\mathrm{M}=\mathrm{Li}$ or $\mathrm{Na}$, were synthesized according to the following reaction:

$$
\begin{aligned}
& y / 2 \mathrm{M}_{2} \mathrm{WO}_{4}+x / 2 \mathrm{~K}_{2} \mathrm{WO}_{4}+[1-(x+y)] \mathrm{WO}_{3} \\
&+(x+y) / 2 \mathrm{WO}_{2} \rightarrow \mathrm{M}_{y} \mathrm{~K}_{x} \mathrm{WO}_{3} .
\end{aligned}
$$

Finely ground, intimate mixtures of the dried reactants with $0.02<x<0.52$ and $0<y<0.5$ were heated at various temperatures between $500^{\circ} \mathrm{C}$ and $850^{\circ} \mathrm{C}$ for periods ranging from 3 days to 3 weeks in evacuated sealed silica tubes. The longer heating times were used to confirm results obtained following short heat treatments. In some cases the samples were kept in small gold tubes inside the silica tubes, particularly for those compositions where slight attack on the silica had been observed. No significant difference in the results was noticed, however.

The products obtained were not always single phases. They could contain, in addition to bronze phases, non-bronze phases, unreacted materials and even, in some cases, crystallized silica from the reaction tube. Alkali tungsten bronzes are normally chemically very inert ${ }^{12}$ and advantage can be taken of this property for their purification. The following procedure had previously been used successfully: ${ }^{13}$ The samples were treated in a platinum crucible successively with (i) boiling water, (ii) boiling $40 \%$ hydrofluoric acid and (iii) boiling $5 \%$ sodium hydroxide solution, with thorough washing with water in between. Finally, the bronzes were washed with ethanol and dried in air at $100^{\circ} \mathrm{C}$. However, with the Li-doped bronzes the above procedure lead to leaching out of lithium, as discussed below.

The samples prepared were examined in an optical microscope before and after the purification procedure. The various bronze phases could then be identified by their characteristic colour. ${ }^{1} \mathrm{X}$-ray powder patterns were also recorded before and after the purification procedure, using a GuinierHägg focusing camera and $\mathrm{CuK \alpha} \alpha_{1}$ $(\lambda=1.540598 \AA) \quad$ radiation. $\mathrm{KCl}$ $(a=6.292948 \AA)$ or Si $(a=5.430879 \AA)$ were used as internal standards whenever cell parameters were to be refined. The films were evaluated using a computerized film scanner ${ }^{14}$ and unit cell parameters were refined by least-squares analysis. The calculated standard deviations were in the range $2-5 \cdot 10^{-5}$ and were experimentally verified by repeated recording and measurement of some of the patterns.

An electron microscope (Siemens ELMISKOP 102 with a tilt-lift stage, operated at $125 \mathrm{kV}$ ) was also used for identification of the various bronze phases from their characteristic electron diffraction patterns. Secondary electron micrographs of bronze crystals were taken with a JSM-U3 type scanning electron microscope at $25 \mathrm{kV}$.

The bronzes were analysed for lithium and potassium by atomic absorption spectrometry, as described earlier. ${ }^{13}$ The observed potassium content in the bronzes agreed closely with the initial gross composition. However, the lithium content found was much too low in the "purified" bronze samples.

The initial step in the analysis procedure was oxidation of the sample in air at $600^{\circ} \mathrm{C}$. In order to investigate whether the discrepancy was due to loss of some volatile lithium-containing species during this high temperature treatment, an alternative method of oxidation was tested. The samples were fused with sodium nitrate $\left(\mathrm{Li}_{y} \mathrm{~K}_{x} \mathrm{WO}_{3}: \mathrm{NaNO}_{3}=1: 3\right)$ for a few minutes in an open silica tube over a flame. The solidified clear melt was then dissolved in water which had been made slightly alkaline and lithium was determined by atomic absorption spectrometry. This method is much quicker than that previously employed. The lithium analyses were still very low, however. When "unpurified", apparently single-phase samples were analysed, however, good agreement with the gross composition was obtained. The implications of this are discussed further below.

\section{Results}

The results of the phase analysis are summarized in Fig. 1. With the preparation carried out in closed tubes the gross composition of the samples should remain constant. The composition of a phase is then known whenever it can be proved to be the only phase in the sample. However, for polyphasic samples, analysis of the bronze phase is necessary for $x$ and $y$ to be known with certainty. As mentioned above, the analysis made of the purified bronzes gave values close to those expected for potassium, but the lithium content was consistently much too low (typically about $20 \%$ of the expected value). Analysis of "unpu- 


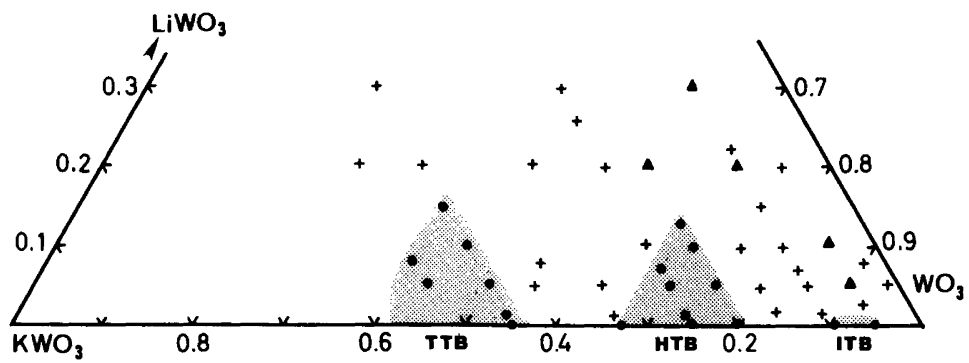

Fig. 1. Compositions investigated in the $\mathrm{Li}_{y} \mathrm{~K}_{x} \mathrm{WO}_{3}$ system. $=$ single phase; $+=$ two major phases observed; $\boldsymbol{\Delta}=$ three major phases. Shaded areas indicate suggested single-phase regions.

ritied", apparently single phase samples, however, gave good agreement. It thus appeared that lithium was removed from the samples during the purification process. Tests showed that treatment with water alone was sufficient to leach out a significant proportion of lithium.

In view of the high chemical stability of other alkali metal-containing bronzes this result was rather surprising but could be explained in two ways: (i) lithium is present in the bronze phase but is easily removed or replaced, or (ii) all or most of the lithium is present in an additional, soluble, possibly amorphous phase which is not detected by microscopic examination (optical and SEM) or in the X-ray powder patterns. The results presented below suggest that explanation (i) is the more probable.

Intergrowth tungsten bronzes, ITB. Attempts to prepare pure lithium or sodium ITB at $850^{\circ} \mathrm{C}$ were unsuccessful. This is not surprising in view of the non-existence of the corresponding HTB phase at this temperature. Moreover, single phases of mixed lithium-potassium ITB could not be prepared. The samples obtained were always mixtures of ITB, HTB and $\mathrm{WO}_{3}$. Of 52 crystals from various lithium-doped samples investigated in the transmission electron microscope, 17 ITB, $11 \mathrm{HTB}$ and $24 \mathrm{WO}_{3}$ crystals were found. Since there was no single-phase lithium-potassium ITB, analysis for lithium could not be performed by atomic absorption spectrometry. We tried to determine lithium in individual crystals by means of electron energy loss spectrometry (EELS). However, this was unsuccessful due to the presence of tungsten in the sample which gave high background noise and thereby concealed any lithium signal.
Hexagonal tungsten bronzes, HTB. A mixed sodium-potassium HTB phase could not be prepared. The products obtained for $x=0.2$ and $x+y<0.4$ after heating at $850^{\circ} \mathrm{C}$ were always mixtures of TTB and HTB.

With lithium, optical microscope examination and X-ray powder patterns revealed that pure, homogeneous HTB phases could be prepared for $x>0.19$ and $x+y<0.33$ (Fig. 1). Samples pre-
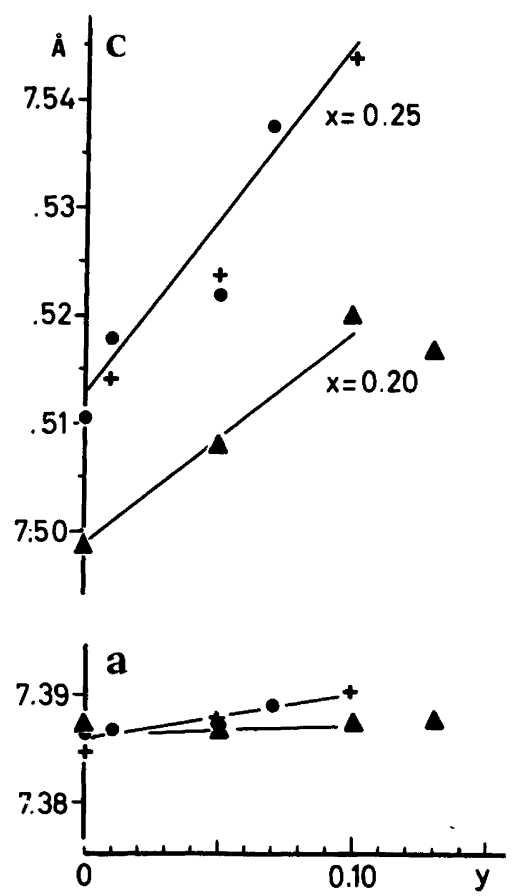

Fig. 2. Hexagonal lattice parameters for $\mathrm{Li}_{y} \mathrm{~K}_{x} \mathrm{WO}_{3}$ HTB as a function of $y$. and $\boldsymbol{\Delta}=$ prepared at 850 ${ }^{\circ} \mathrm{C} ;+=$ prepared at $500^{\circ} \mathrm{C}$. 

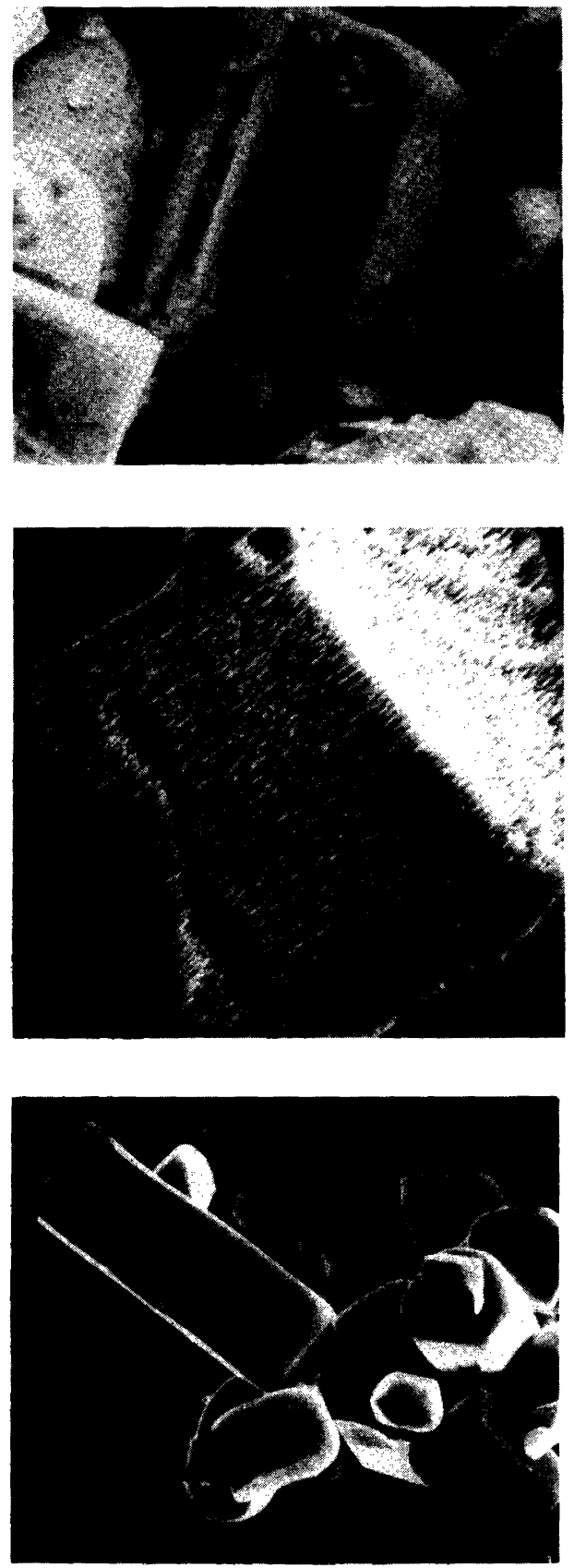

Fig. 3. SEM images of (a) $\mathrm{Li}_{0.1} \mathrm{~K}_{0.2} \mathrm{WO}_{3}$ without "purification"; (b) $\mathrm{Li}_{0.1} \mathrm{~K}_{0.25} \mathrm{WO}_{3}$ after "purification" in $40 \% \mathrm{HF}$ and $5 \% \mathrm{NaOH}$; (c) pure K-HTB after the same treatment. Same magnification in all images. Note the deterioration of the crystals in (b) but not in (c). pared outside these limits contained a mixture of bronze (PTB, HTB, TTB) and non-bronze phases (tungstates).

The $a$ parameter for $\mathrm{Li}_{y} \mathrm{~K}_{x} \mathrm{WO}_{3}(\mathrm{HTB})$ is practically constant with $y$, whereas $c$ increases, as seen in Fig. 2.

SEM images of lithium-potassium HTB samples showed that the crystals were attacked during the purification process (Fig. 3).

Tetragonal tungsten bronzes, TTB. Pure homogeneous tetragonal phases (TTB) were obtained with both lithium and sodium for $x>0.45$ and $x+y<0.6$ (Fig. 1). K-TTB with $x>0.48$ is difficult or impossible to prepare by solid state reaction in closed ampoules at $500^{\circ} \mathrm{C}$ or higher. ${ }^{1}$ However, $\mathrm{Li}-\mathrm{K}-\mathrm{-TTB}$ with $x+y>0.48$ were obtained at $800^{\circ} \mathrm{C}$ in the present investigation (Fig. 1). These mixed TTB phases decompose above $900^{\circ} \mathrm{C}$, as does pure $\mathrm{K}$-TTB. ${ }^{1}$

Both the $a$ and $c$ parameters for $\mathrm{Li}_{y} \mathrm{~K}_{x} \mathrm{WO}_{3}$ (TTB) are practically constant with $(x+y)$ (Fig. 4). Addition of sodium to potassium TTB gives rise to a slight increase in both these cell parameters (Fig. 4).

\section{Discussion}

The structure of intergrowth tungsten bronze consists of 2-dimensionally infinite slabs of HTBtype, interleaved with slabs of slightly distorted $\mathrm{WO}_{3}$-type, which together form a coherent 3-dimensional framework of $\mathrm{WO}_{3}$ composition. ${ }^{15}$ The (heavy) alkali metal atoms are found in the hexagonal tunnels in the HTB-type slabs. ${ }^{15,16}$ The widths of the two structure elements can vary, thereby forming different members of the ITB structure family.

It is interesting to note that very little or no lithium can be incorporated into the K-ITB phase, at least at the high temperatures used in our experiments. There is evidence ${ }^{17,18}$ that the HTB-type tunnel sites in the $\mathrm{K}$ - and Cs-ITB phases are only partially occupied, and there should thus be room for lithium there. Moreover, the $\mathrm{WO}_{3}$-type slabs might theoretically be able to accommodate a considerable amount of lithium, thus forming a PTB-type bronze. Apparently this does not occur in solid state reactions at high temperatures. It remains to be studied whether lithium can be intercalated into ITB phases at lower temperatures by other means. 

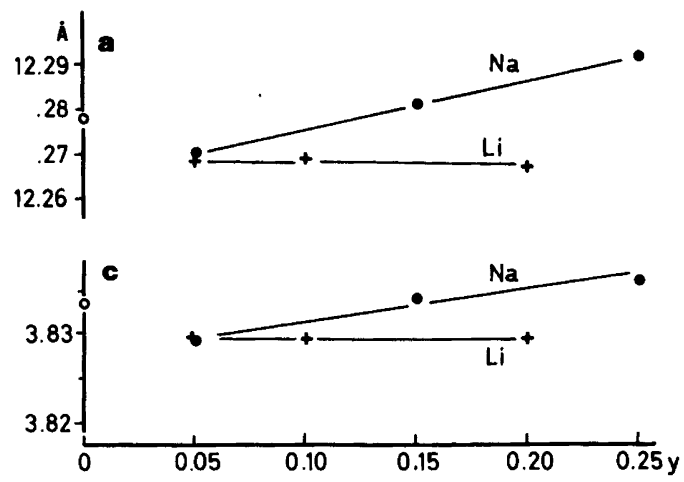

Fig. 4. Tetragonal lattice parameters for $\mathrm{M}_{y} \mathrm{~K}_{0.45} \mathrm{WO}_{3}$ $(\mathrm{M}=\mathrm{Li}$ or $\mathrm{Na})$ vs. $y$. Unfilled circles are values from Ref. 1.

Our syntheses at the hexagonal tungsten bronze compositions indicate that lithium can form a mixed alkali metal HTB phase with potassium, but only if enough potassium is present to make the pure K-HTB phase stable per se. The maximum amount of alkali metal that we have been able to introduce corresponds approximately to full occupancy of the hexagonal tunnel sites, i.e. $x+y=1 / 3$. This is at variance with the results of Banks and Goldstein, ${ }^{3}$ who found that $x+y$ could be as high as 0.51 for $\mathrm{Li}-\mathrm{K}-\mathrm{HTB}$ prepared at $500^{\circ} \mathrm{C}$. When we prepared samples with $x=0.25$ and $y=0.20$ we obtained a two-phase mixture of HTB and TTB after reaction at $500^{\circ} \mathrm{C}$ for 3 weeks.

Raistrick et al. ${ }^{9}$ prepared $\mathrm{Li}-\mathrm{K}-\mathrm{HTB}$ with $x=0.3$ and $y<0.36$ by electrochemical intercalation. An even higher lithium content, $y=0.67$, has been reached by Cheng et al. ${ }^{10,11}$ by treatment of $\mathrm{K}_{0.33} \mathrm{WO}_{3}$ with $n$-butyl lithium. However, these experiments were made at room temperature, where the stability range cannot be expected to be the same as at high temperatures.

Our results differ from those of Banks and Goldstein $^{3}$ also with respect to the reactivity of the lithium-containing phases. These authors report that they could treat the bronze samples with rather strong (48\%) hydrofluoric acid and sodium carbonate solution without any noticeable loss of lithium, whereas we found a substantial lithium loss even in pure, distilled water and observed marked deterioration of the crystals after such treatment.

It is to be expected that treatment in an oxidiz- ing environment should lead to increased solubility. We carried out experiments in which we tried to avoid oxidizing agents as far as possible, for example by using boiled water under nitrogen, but found no difference. It might also be suspected that our samples contained a slight excess of oxygen as prepared, despite our efforts to keep the stoichiometry correct. Fully oxidized potassium polytungstates with a structure similar to that of HTB are, in fact, known. ${ }^{19,20}$ However, the oxygen excess, if any, must be quite small and if this were the origin of the large difference in reactivity it would be remarkable. A further study of this problem is planned.

The unit cell dimensions give clear evidence of the formation of mixed $\mathbf{L i}+\mathrm{K}$ HTB bronze. There is a marked increase in the length of the $c$ axis with increasing Li content. The $a$ axis, on the other hand, changes very little. Our values for $c$ are approximately $0.5 \%$ lower than those reported by Banks and Goldstein. ${ }^{3}$ For a sample with $x=0.24$ they found a rather sharp increase in $c$ from $y=0$ to 0.04 and a constant value for higher lithium contents. They measured the lattice parameters with a diffractometer, but since no further details are given, an analysis of the cause of this discrepancy cannot be made. Lithium-containing samples which have been "purified" show $c$-values that are about $0.1 \%$ lower than those for the corresponding "unpurified" phase. This is consistent with the idea that part of the lithium is actually leached out of the crystals by this treatment.

It is not possible at the present stage to tell where the Li atoms are located in the HTB structure. Since we find that the total alkali content does not exceed $(x+y)=$ approx. 1/3 at the preparation temperatures used by us, it seems reasonable to assume that all alkali metal atoms go into the normal hexagonal tunnel sites. However, only a very careful structure analysis can verify this.

The results obtained for TTB indicate that both the pentagonal and the tetragonal tunnel sites are available for the alkali atoms, corresponding to a maximum alkali content of $x+y=0.60$. Lithium and sodium are likely to fit better than potassium into the tetragonal, perovskite-type cages. A single crystal investigation $^{21}$ has shown that potassium prefers the larger pentagonal tunnels in pure K-TTB, and potassium contents corresponding to high occupancy 
of the tetragonal tunnels are very difficult to attain. ${ }^{1}$ However, the actual sites of the different alkali metal atoms in the mixed bronze remain to be determined experimentally.

Acknowledgements. The stay of A.H. at the Arrhenius Laboratory was made possible by the International Seminar in Chemistry of Uppsala University. This investigation forms part of a research program supported by the Swedish Natural Science Research Council.

\section{References}

1. Hussain, A. Acta Chem. Scand., Ser. A32 (1978) 479.

2. Hussain, A. Chem. Commun. Univ. Stockholm 1978, No. 2.

3. Banks, E. and Goldstein, A. Inorg. Chem. 7 (1968) 966.

4. Utsumi, S. and Fujieda, S. Bull. Chem. Soc. Jpn. 43 (1970) 1714.

5. Fujicda, S. Natural Sci. Rep., Ochanomizu Univ., 25 (1974) 35.

6. Drobasheva, T. I. and Skoropad, T.S. Izv. Akad. Nauk SSSR, Neorg. Mater. 14 (1978) 305.
7. Drobasheva, T. I., Skoropad, T. S. and Bukhalova, G. A. Zh. Neorg. Khim. 22 (1977) 1961.

8. Drobasheva, T. I. and Skoropad, T.S. Zh. Neorg. Khim. 23 (1978) 171.

9. Raistrick, I. D., Mark, A. J. and Huggins, R. A. Solid State Ionics 5 (1981) 351.

10. Cheng, K. H. and Whittingham, M.S. Solid State Ionics $I$ (1980) 151.

11. Cheng, K. H., Jacobson, A. J. and Whittingham, M. S. Solid State Ionics 5 (1981) 355.

12. Magnéli, A. and Blomberg, B. Acta. Chem. Scand. 5 (1951) 372.

13. Hussain, A. and Kihlborg, L. Anal. Chim. Acta 90 (1977) 283.

14. Johansson, K.-E., Palm, T. and Werner, P.-E. J. Phys. E 13 (1980) 1289.

15. Hussain, A. and Kihlborg, L. Acta Crystallogr., Sect. A32 (1976) 551.

16. Hussain, A. Chem. Scr. 11 (1977) 224.

17. Kihlborg, L. Chem. Scr. 14 (1979) 187.

18. Fernandez, M. and Kihlborg, L. To be published.

19. Klug, A. Chem. Commun. Univ. Stockholm 1977, No. 7.

20. Klug, A. Mater. Res. Bull. 12 (1977) 837.

21. Kihlborg, L. and Klug, A. Chem. Scr. 3 (1973) 207.

Received July 8, 1986. 